\title{
Correlation Results for a Mass Loaded Vehicle Panel Test Article Finite Element Models and Modal Survey Tests
}

\author{
Rumaasha Maasha ${ }^{1}$ \\ NASA/Marshall Space Flight Center, Huntsville. AL, 35612 \\ Robert L. Towner ${ }^{2}$ \\ Jacobs Engineering, ESTS Group, Huntsville. AL, 35806
}

\begin{abstract}
High-fidelity Finite Element Models (FEMs) were developed to support a recent test program at Marshall Space Flight Center (MSFC). The FEMs correspond to test articles used for a series of acoustic tests. Modal survey tests were used to validate the FEMs for five acoustic tests (a bare panel and four different mass-loaded panel configurations). An additional modal survey test was performed on the empty test fixture (orthogrid panel mounting fixture, between the reverb and anechoic chambers). Modal survey tests were used to test-validate the dynamic characteristics of FEMs used for acoustic test excitation. Modal survey testing and subsequent model correlation has validated the natural frequencies and mode shapes of the FEMs. The modal survey test results provide a basis for the analysis models used for acoustic loading response test and analysis comparisons
\end{abstract}

$\begin{array}{ll} & \\ \text { COMAC } & =\text { Coordinate Modal Assurance Criteria } \\ \text { ESTS } & =\text { Engineering, Science, and Technical Services } \\ F E M & =\text { Finite Element Model } \\ F R F & =\text { Frequency Response Function } \\ I U & =\text { Instrument Unit } \\ M A C & =\text { Modal Assurance Criteria } \\ M S F C & =\text { Marshall Space Flight Center } \\ \text { RSS } & =\text { Root Sum Squared } \\ X O R & =\text { Cross-Orthogonality }\end{array}$

\section{Introduction}

$\mathrm{M}$ odal survey testing was performed to measure the mode shapes, natural frequencies and modal damping factors of an orthogrid panel. NASTRAN Finite Element Models (FEMs) were correlated to obtain adequate agreement with modal survey test results, providing a basis for test validation of the FEM dynamic characteristics for subsequent acoustic response test and analysis comparisons. Model correlation was performed on a sequential/progressive basis, from simpler (less built-up configurations) to more complex test configurations. FEM of the test fixture was correlated to empty test fixture configuration (and unchanged for subsequent test configurations), followed by model correlation of the orthogrid panel for the bare panel configuration, followed by model correlation for four mass-loaded test configurations (without modifying the FEMs of the orthogrid panel or test fixture that had been correlated in the previous test configurations). Test validation of the analysis model dynamic characteristics for specific acoustic response test configurations eliminates FEM uncertainties from acoustic response test and analysis comparisons.

\section{Modal Survey Testing}

A series of six modal survey tests were performed at the MSFC Structural Dynamics Test Branch's Acoustic Test Laboratory. In-Situ testing was performed in the acoustic test facility, with tri-axial accelerometers mounted on

${ }^{1}$ Loads and Dynamics Engineer, MSFC/EV31, Huntsville, AL, AIAA Member.

${ }^{2}$ Dynamics \& Loads Tech Fellow, Jacobs Engineering, 1500 Perimeter Parkway, Huntsville, AL, AIAA Member. 
the test articles. A total of 32 tri-axial accelerometers were used for the empty fixture test, 77 tri-axial accelerometers were used for the bare panel test, and 85 tri-axial accelerometers were used for each of the massloaded panel configurations. Impact hammer excitation was used to excite the test articles. Input and response data was acquired and Frequency Response Functions (FRFs), coherence functions, and input/response auto-powers and cross-powers were generated for each test configuration. Modal Indicator Functions were used to identify modes for each of the six test configurations, and experimental frequencies, mode shapes, and modal damping factors were generated. Modal parameter identification was used to calculate modes for each test configuration for a frequency range of interest (modes up to $250 \mathrm{~Hz}$ to $350 \mathrm{~Hz}$, depending on the test configuration).

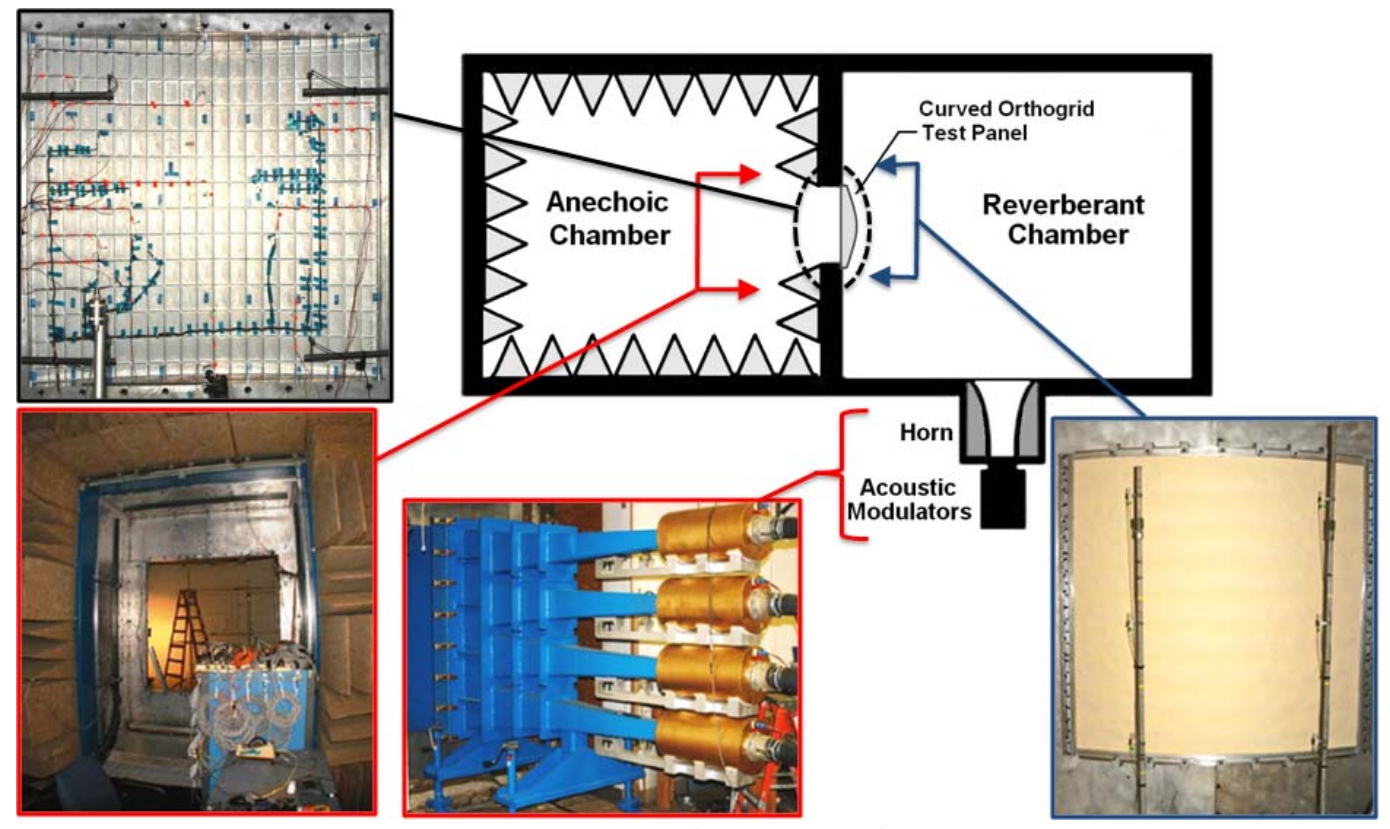

Figure 1. Modal Survey Test Set-up.

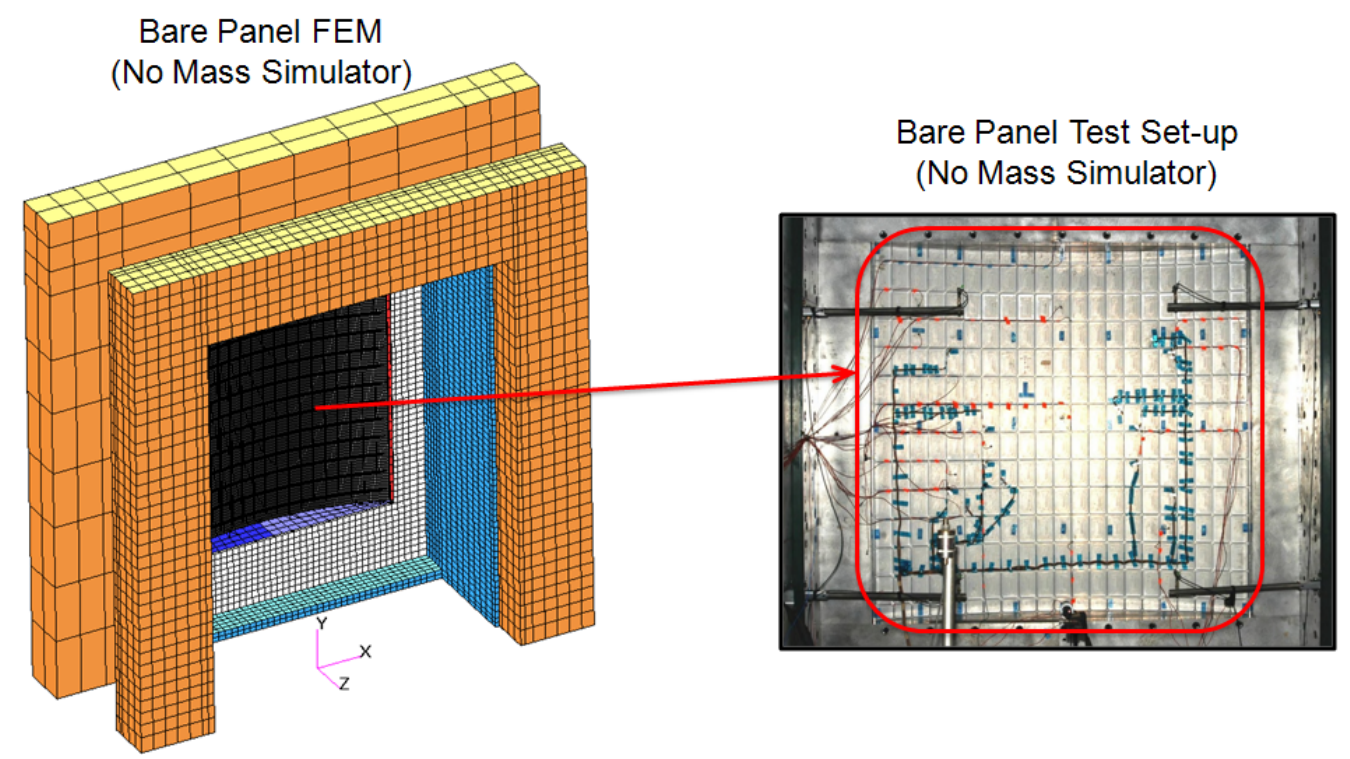

Figure 2. Bare Panel Test Set-Ud 
Small Mass Simulator-Aft Skirt (AS)
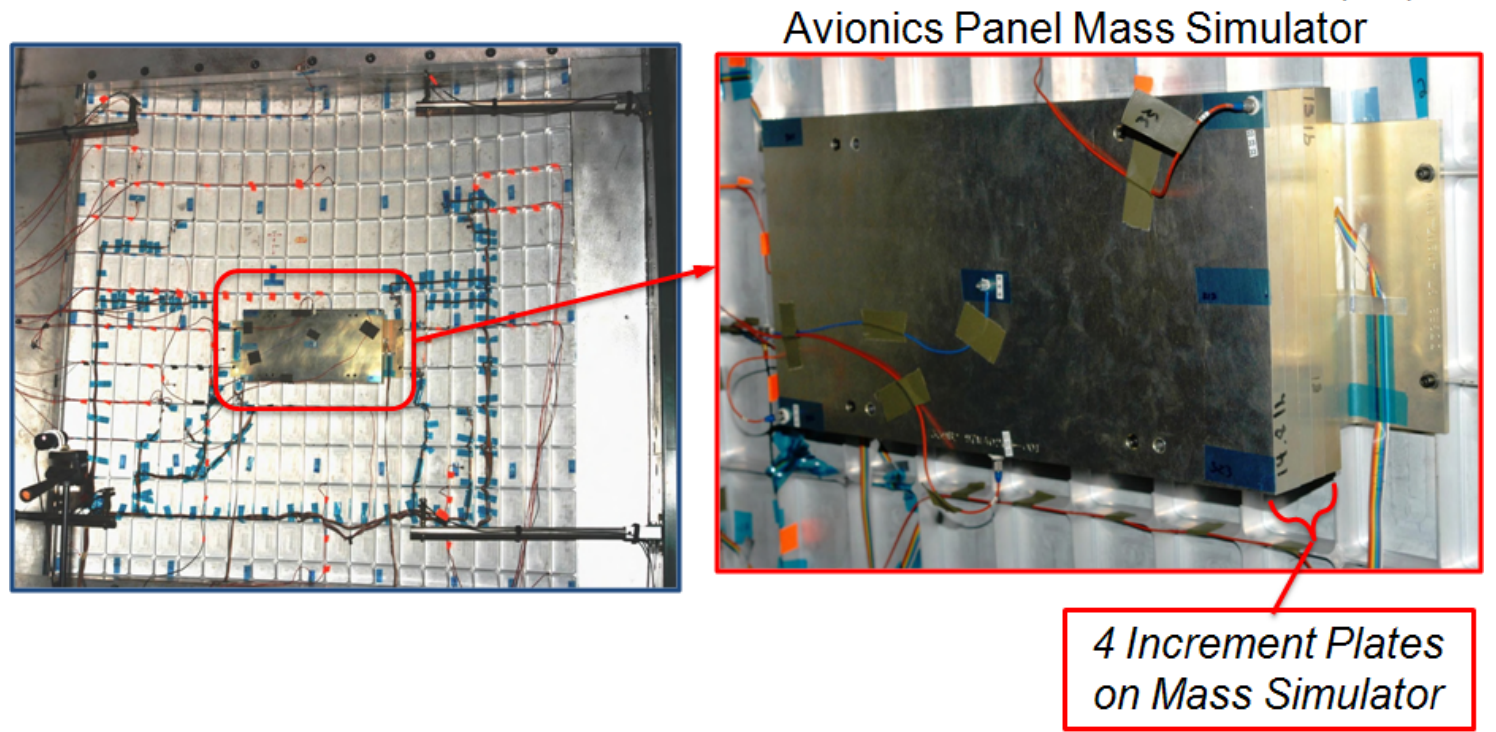

Figure 3. Aft Skirt Avionics Panel Test Set-Up

Large Mass Simulator-Instrument Unit (IU) Avionics Panel Mass Simulator
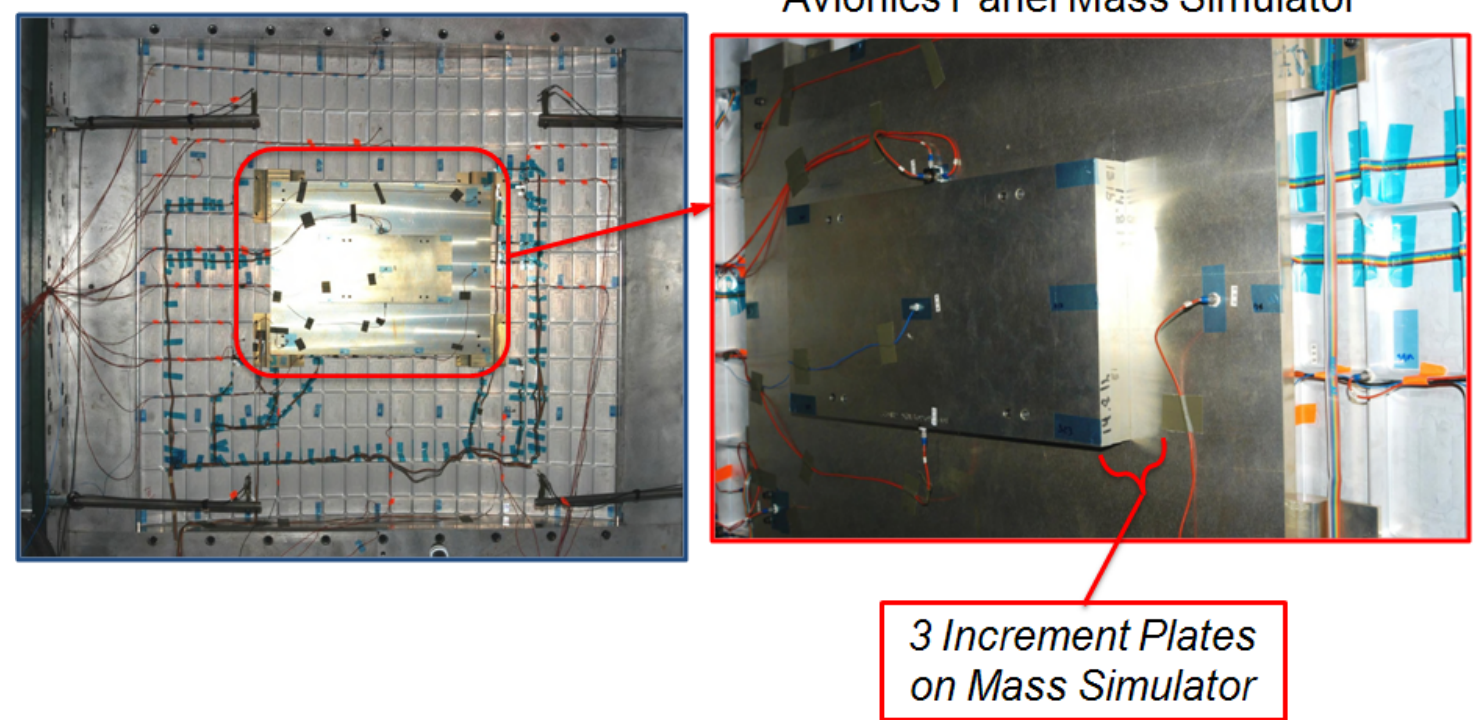

Figure 4. Instrument Unit (IU) Avionics Panel Test Set-Up 


Empty Fixture
Test Modes
\begin{tabular}{|c|c|}
\hline $\begin{array}{c}\text { Mode } \\
\text { No. }\end{array}$ & $\begin{array}{c}\text { Freq } \\
\mathrm{Hz}\end{array}$ \\
\hline 1 & 119.25 \\
\hline 2 & 123.86 \\
\hline 3 & 138.32 \\
\hline 4 & 169.64 \\
\hline 5 & 238.73 \\
\hline 6 & 266.68 \\
\hline 7 & 345.51 \\
\hline
\end{tabular}

Bare Panel
Test Modes
\begin{tabular}{|c|c|}
\hline $\begin{array}{c}\text { Mode } \\
\text { No. }\end{array}$ & $\begin{array}{c}\text { Freq, } \\
\mathrm{Hz}\end{array}$ \\
\hline 1 & 101.21 \\
\hline 2 & 110.01 \\
\hline 3 & 150.75 \\
\hline 4 & 159.82 \\
\hline 5 & 186.64 \\
\hline 6 & 207.79 \\
\hline 7 & 208.64 \\
\hline 8 & 218.95 \\
\hline 9 & 242.66 \\
\hline 10 & 251.70 \\
\hline 11 & 281.13 \\
\hline 12 & 291.97 \\
\hline 13 & 319.69 \\
\hline
\end{tabular}

AS Mass Simulator with 0 Increment Plates Test Modes

\begin{tabular}{|c|c|}
\hline $\begin{array}{c}\text { Mode } \\
\text { No. }\end{array}$ & $\begin{array}{c}\text { Freq, } \\
\mathrm{Hz}\end{array}$ \\
\hline 1 & 84.77 \\
\hline 2 & 95.27 \\
\hline 3 & 142.87 \\
\hline 4 & 160.59 \\
\hline 5 & 163.36 \\
\hline 6 & 172.05 \\
\hline 7 & 173.99 \\
\hline 8 & 186.69 \\
\hline 9 & 204.83 \\
\hline 10 & 213.84 \\
\hline 11 & 231.32 \\
\hline 12 & 249.94 \\
\hline 13 & 293.94 \\
\hline
\end{tabular}

AS Mass Simulator with 3

IU Mass Simulator with 0

IU Mass Simulator with 3 Increment Plates Test Modes Increment Plates Test Modes Increment Plates Test Modes

\begin{tabular}{|c|c|}
\hline $\begin{array}{c}\text { Mode } \\
\text { No. }\end{array}$ & $\begin{array}{c}\text { Freq, } \\
\mathrm{Hz}\end{array}$ \\
\hline 1 & 64.56 \\
\hline 2 & 67.91 \\
\hline 3 & 91.88 \\
\hline 4 & 154.08 \\
\hline 5 & 167.94 \\
\hline 6 & 173.91 \\
\hline 7 & 198.10 \\
\hline 8 & 212.77 \\
\hline 9 & 227.29 \\
\hline 10 & 238.13 \\
\hline 11 & 247.44 \\
\hline 12 & 255.08 \\
\hline 13 & 293.94 \\
\hline
\end{tabular}

\begin{tabular}{|c|c|}
\hline $\begin{array}{c}\text { Mode } \\
\text { No. }\end{array}$ & $\begin{array}{c}\text { Freq, } \\
\mathrm{Hz}\end{array}$ \\
\hline 1 & 71.05 \\
\hline 2 & 73.04 \\
\hline 3 & 118.07 \\
\hline 4 & 157.34 \\
\hline 5 & 165.09 \\
\hline 6 & 194.95 \\
\hline 7 & 228.67 \\
\hline 8 & 241.93 \\
\hline 9 & 257.31 \\
\hline
\end{tabular}

\begin{tabular}{|c|c|}
\hline $\begin{array}{c}\text { Mode } \\
\text { No. }\end{array}$ & $\begin{array}{c}\text { Freq, } \\
\mathrm{Hz}\end{array}$ \\
\hline 1 & 66.40 \\
\hline 2 & 68.03 \\
\hline 3 & 114.97 \\
\hline 4 & 156.96 \\
\hline 5 & 174.50 \\
\hline 6 & 198.61 \\
\hline 7 & 234.31 \\
\hline 8 & 249.00 \\
\hline
\end{tabular}

Figure 6. Summary of Modal Survey Test Modes

\section{Test and Analysis Model Correlation}

Test results were compared to the FEM predictions, and the analysis models were updated until acceptable correlation was obtained. Detailed review of test data identified differences from analysis results, and indicated potential sources of these differences. Updates were made to the test fixture FEM and its boundary conditions, incorporating CAD geometry of the test fixture (structure supporting the orthogrid panel between the reverberant and anechoic chambers). FEMs of the orthogrid panel and mass simulators/increment plates were adequate based on the pre-test analysis model(s). Model correlation was demonstrated using a Matlab program to compare test and analysis modes using frequency difference, Modal Assurance Criteria (MAC), Cross-Orthogonality (XOR), and Coordinate Modal Assurance Criteria (COMAC), along with side-by-side test and analysis mode shapes (static and animated mode shapes).

The MAC compares two vectors by calculating the dot or scalar product and dividing by the magnitude of the vectors. This calculation gives the cosine of the angle between the two vectors, yielding a numerical indicator of 1.0 when the angle is 0 or 180 -degrees, and 0.0 when the vectors are orthogonal. 


$$
\left.\{M A C\}=\frac{\left.\left[\left\{P_{\text {anal }}\right\}\right\}^{T} \times\left\{P H I_{\text {test }}\right\}\right]^{2}}{\left[\left\{P H I_{\text {anal }}\right\}^{T} \times\left\{P H I_{\text {anal }}\right\}\right] \times\left[\left\{P H I_{\text {test }}\right\}^{T} \times\left\{P H I_{\text {test }}\right\}\right.}\right]
$$

Where: $\left\{\mathrm{PHI}_{\text {anal }}\right\}=$ Analysis Eigenvector Matrix

$\left\{\mathrm{PHI}_{\text {test }}\right\}=$ Test Eigenvector Matrix

Another accepted numerical technique for comparing a pair of mode shapes includes the Cross-Orthogonality:

$$
\{X O R\}=\left\{P H I_{\text {test }}\right\}^{T} \times\{M A A\} \times\left\{P H I_{\text {anal }}\right\}
$$

Where: $\left\{\mathrm{PHI}_{\text {anal }}\right\}=$ Analysis Eigenvector Matrix

$\left\{\mathrm{PHI}_{\text {test }}\right\}=$ Test Eigenvector Matrix

$\{$ MAA $\}=$ Reduced Analytical Mass Matrix

The XOR value between two mode shape vectors can vary from -1.0 to +1.0 , with values of -1.0 or +1.0 showing that the mode shapes are perfectly correlated, and a value of 0.0 indicating that the vectors are uncorrelated.

\section{Final Correlation Results}

Test-validated FEMs for the six test configurations are preferable for use in acoustic response analyses, based on test-validated dynamic characteristics in the 0 to $250-350 \mathrm{~Hz}$ frequency range.

\begin{tabular}{|c|c|c|c|c|c|c|}
\hline $\begin{array}{c}\text { TEST } \\
\text { MODE }\end{array}$ & $\begin{array}{l}\text { TEST } \\
\text { FREQ }\end{array}$ & $\begin{array}{c}\text { FEM } \\
\text { MODE }\end{array}$ & $\begin{array}{l}\text { FEM } \\
\text { FREQ }\end{array}$ & MAC & $\begin{array}{l}\text { CROSS- } \\
\text { ORTHOG }\end{array}$ & $\begin{array}{l}\text { FREQ } \\
\text { DIFF }\end{array}$ \\
\hline 1 & 119.25 & 1 & 110.41 & 0.92 & -0.87 & $-7.4 \%$ \\
\hline 2 & 123.86 & 2 & 129.27 & 0.88 & 0.94 & $4.4 \%$ \\
\hline 3 & 138.32 & 4 & 158.12 & 0.60 & 0.70 & $14.3 \%$ \\
\hline 4 & 169.64 & 3 & 149.49 & 0.73 & 0.93 & $-11.9 \%$ \\
\hline 5 & 238.73 & 14 & 279.29 & 0.71 & -0.73 & $17.0 \%$ \\
\hline 6 & 266.68 & 14 & 279.29 & 0.68 & 0.64 & $4.7 \%$ \\
\hline 7 & 345.51 & 19 & 358.12 & 0.35 & -0.44 & $3.7 \%$ \\
\hline
\end{tabular}

Table 1. Empty Fixture Test and Analysis Correlation Summary

- Typical side-by-side test and analysis mode shapes shown for the first two mode pairs for the empty fixture test configuration.

\begin{tabular}{|c|c|c|c|c|c|c|}
\hline $\begin{array}{c}\text { TEST } \\
\text { MODE }\end{array}$ & $\begin{array}{l}\text { TEST } \\
\text { FREQ }\end{array}$ & $\begin{array}{c}\text { FEM } \\
\text { MODE }\end{array}$ & $\begin{array}{c}\text { FEM } \\
\text { FREQ }\end{array}$ & MAC & $\begin{array}{l}\text { CROSS- } \\
\text { ORTHOG }\end{array}$ & $\begin{array}{c}\text { FREQ } \\
\text { DIFF }\end{array}$ \\
\hline 1 & 101.21 & 1 & 97.20 & 0.96 & -0.99 & $-4.0 \%$ \\
\hline 2 & 110.01 & 2 & 106.67 & 0.95 & 0.97 & $-3.0 \%$ \\
\hline 3 & 150.75 & 5 & 143.04 & 0.93 & -0.91 & $-5.1 \%$ \\
\hline 4 & 159.82 & 7 & 155.33 & 0.94 & -0.98 & $-2.8 \%$ \\
\hline 5 & 186.64 & 9 & 181.98 & 0.90 & 0.90 & $-2.5 \%$ \\
\hline 6 & 207.79 & 10 & 198.37 & 0.86 & 0.92 & $-4.5 \%$ \\
\hline 7 & 208.64 & 11 & 202.65 & 0.51 & -0.54 & $-2.9 \%$ \\
\hline 8 & 218.95 & 14 & 211.19 & 0.92 & 0.98 & $-3.6 \%$ \\
\hline 9 & 242.66 & 17 & 233.96 & 0.75 & -0.86 & $-3.6 \%$ \\
\hline 10 & 251.70 & 18 & 243.28 & 0.91 & -0.97 & $-3.4 \%$ \\
\hline 11 & 281.13 & 21 & 268.45 & 0.39 & 0.60 & $-4.5 \%$ \\
\hline 12 & 291.97 & 26 & 283.18 & 0.75 & -0.68 & $-3.0 \%$ \\
\hline 13 & 319.69 & 30 & 307.89 & 0.88 & 0.90 & $-3.7 \%$ \\
\hline
\end{tabular}

Table 2. Bare Panel Test and Analysis Correlation Summary

- Typical side-by-side test and analysis mode shapes shown for the first and last mode pairs for the bare panel test configuration.

$\Rightarrow$ - Important modes showing high response for the bare panel acoustic response test. 
Table 3. Aft Skirt Mass Simulator without Increment Plates Test and Analysis Correlation Summary

$\longrightarrow \quad$\begin{tabular}{|c|c|c|r|r|r|r|}
\hline TEST & TEST & FEM & FEM & & CROSS- & FREQ \\
\hline MODE & FREQ & MODE & FREQ & MAC & ORTHOG & DIFF \\
\hline 1 & 84.77 & 1 & 82.92 & 0.97 & 0.99 & $-2.2 \%$ \\
\hline 2 & 95.27 & 2 & 94.76 & 0.98 & 0.99 & $-0.5 \%$ \\
\hline 3 & 142.87 & 4 & 138.35 & 0.87 & -0.78 & $-3.2 \%$ \\
\hline 4 & 160.59 & 7 & 156.49 & 0.93 & -0.97 & $-2.6 \%$ \\
\hline 5 & 163.36 & 8 & 162.62 & 0.85 & -0.83 & $-0.5 \%$ \\
\hline 6 & 172.05 & 8 & 162.62 & 0.81 & -0.67 & $-5.5 \%$ \\
\hline 7 & 173.99 & 10 & 170.23 & 0.91 & 0.91 & $-2.2 \%$ \\
\hline 8 & 186.69 & 11 & 188.00 & 0.95 & -0.99 & $0.7 \%$ \\
\hline 9 & 204.83 & 12 & 198.85 & 0.92 & 0.92 & $-2.9 \%$ \\
\hline 10 & 213.84 & 14 & 206.50 & 0.87 & -0.87 & $-3.4 \%$ \\
\hline 11 & 231.32 & 16 & 221.27 & 0.68 & 0.66 & $-4.4 \%$ \\
\hline 12 & 249.94 & 19 & 243.84 & 0.86 & -0.94 & $-2.4 \%$ \\
\hline 13 & 293.94 & 25 & 283.94 & 0.70 & 0.50 & $-3.4 \%$ \\
\hline
\end{tabular}

Important modes showing high response for the AS mass simulator with 0 increment plates acoustic response test.

Table 4. Aft Skirt Mass Simulator with 3 Increment Plates Test and Analysis Correlation Summary

$\Rightarrow$\begin{tabular}{|c|c|c|c|c|c|c|}
\hline TEST & TEST & FEM & FEM & & CROSS- & $\begin{array}{c}\text { FREQ } \\
\text { MIFF }\end{array}$ \\
\hline 1 & 64.56 & 2 & 70.80 & 0.94 & 0.98 & $9.7 \%$ \\
\hline 2 & 67.91 & 1 & 66.27 & 0.97 & 0.99 & $-2.4 \%$ \\
\hline 3 & 91.88 & 3 & 107.38 & 0.86 & 0.97 & $16.9 \%$ \\
\hline 4 & 154.08 & 6 & 147.48 & 0.88 & 0.81 & $-4.3 \%$ \\
\hline 5 & 167.94 & 10 & 168.96 & 0.75 & -0.87 & $0.6 \%$ \\
\hline 6 & 173.91 & 9 & 166.49 & 0.89 & -0.79 & $-4.3 \%$ \\
\hline 7 & 198.10 & 12 & 194.67 & 0.91 & 0.91 & $-1.7 \%$ \\
\hline 8 & 212.77 & 14 & 206.24 & 0.82 & -0.79 & $-3.1 \%$ \\
\hline 9 & 227.29 & 16 & 221.46 & 0.89 & 0.73 & $-2.6 \%$ \\
\hline 10 & 238.13 & 19 & 243.06 & 0.73 & -0.83 & $2.1 \%$ \\
\hline 11 & 247.44 & 18 & 239.41 & 0.88 & -0.87 & $-3.3 \%$ \\
\hline 12 & 255.08 & 20 & 245.51 & 0.60 & 0.67 & $-3.8 \%$ \\
\hline 13 & 293.94 & 26 & 283.97 & 0.81 & -0.81 & $-3.4 \%$ \\
\hline
\end{tabular}

$\Rightarrow$ - Important modes showing high response for the AS mass simulator with 3 increment plates acoustic response test.

Table 5. IU Mass Simulator without Increment Plates Test and Analysis Correlation Summary

\begin{tabular}{|c|c|c|c|c|c|c|}
\hline TEST & TEST & FEM & FEM & & CROSS & FREQ \\
MODE & FREQ & MODE & FREQ & MAC & ORTHOG & DIFF \\
\hline 1 & 71.05 & 1 & 69.08 & 0.96 & 0.98 & $-2.8 \%$ \\
\hline 2 & 73.04 & 2 & 75.68 & 0.87 & 0.97 & $3.6 \%$ \\
\hline 3 & 118.07 & 4 & 121.27 & 0.99 & -1.00 & $2.7 \%$ \\
\hline 4 & 157.34 & 7 & 160.08 & 0.90 & -0.95 & $1.7 \%$ \\
\hline 5 & 165.09 & 8 & 162.93 & 0.80 & -0.90 & $-1.3 \%$ \\
\hline 6 & 194.95 & 11 & 192.65 & 0.85 & -0.89 & $-1.2 \%$ \\
\hline 7 & 228.67 & 14 & 221.85 & 0.87 & -0.86 & $-3.0 \%$ \\
\hline 8 & 241.93 & 17 & 240.07 & 0.77 & 0.89 & $-0.8 \%$ \\
\hline 9 & 257.31 & 18 & 240.70 & 0.49 & -0.63 & $-6.5 \%$ \\
\hline
\end{tabular}

$\Rightarrow$ - Important modes showing high response for the IU mass simulator 
Table 6. IU Mass Simulator without Increment Plates Test and Analysis Correlation Summary

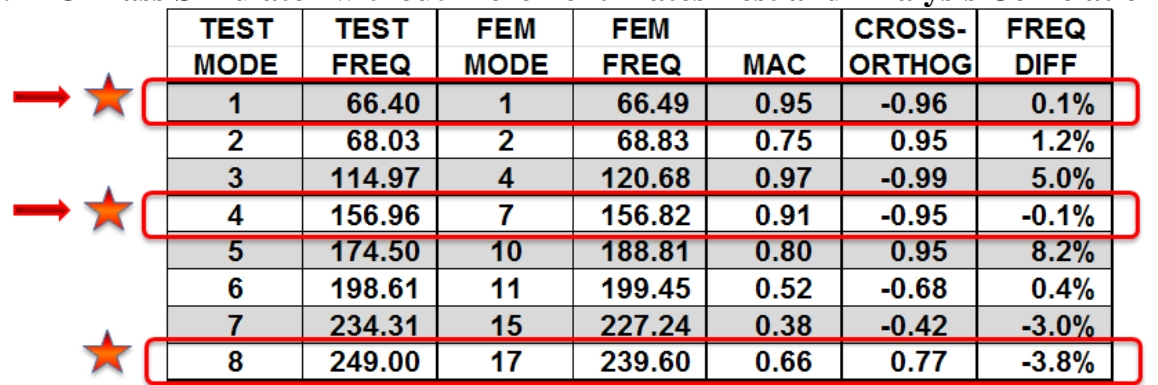

느 - Typical side-by-side test and analysis mode shapes shown for the first and last mode pairs for the IU mass simulator with 3 increment plates.

$\Rightarrow$ - Important modes showing high response for the IU mass simulator

\section{Conclusion}

Six modal survey tests were performed to test-validate the frequencies and mode shapes for analysis models representing the AD01 orthogrid panel acoustic loading test configurations. The test and analysis models were correlated to demonstrate acceptable frequency and mode shape agreement. Correlation demonstrated via percent frequency difference, side-by-side test and analysis mode shape comparisons, Modal Assurance Criteria (MAC), Cross-Orthogonality, and Coordinate Modal Assurance Criteria (COMAC). Test-validated analysis models eliminate uncertainties from acoustic response test and analysis comparisons for the frequency ranges of modal survey tests. These modal survey test-validated FEMs are considered a resource for use in future acoustic load response analyses.

\section{Acknowledgments}

Rumaasha Maasha and Robert Towner thank the Marshall Space Flight Center modal test organization for their outstanding support. The dedicated and capable staff of the ET40 department provided prompt and accommodating support to complete this set of ambitious modal survey tests and evaluate the results.

\section{References}

${ }^{1}$ Ewins, D. J., Modal Testing Theory, Practice, and Application, $2^{\text {nd }}$ ed., Research Studies Press, Ltd., 2000, pp. $422-424$. 